\title{
SNAIL transcription factor increases the motility and invasive capacity of prostate cancer cells
}

\author{
LUIS A. OSORIO, NANCY M. FARFÁN, ENRIQUE A. CASTELLÓN and HÉCTOR R. CONTRERAS \\ Physiology and Biophysics Program, Institute of Biomedical Sciences, \\ Faculty of Medicine, University of Chile, Santiago 8389100, Chile
}

Received January 21, 2015; Accepted October 28, 2015

DOI: $10.3892 / \mathrm{mmr} .2015 .4585$

\begin{abstract}
The incidence and mortality rates of prostate cancer ( $\mathrm{PCa}$ ) are increasing, and $\mathrm{PCa}$ is almost the second-leading cause of cancer-associated mortality in men. During tumor progression, epithelial cells decrease the number of adhesion molecules, change their polarity and position, rearrange their cytoskeleton and increase their migratory and invasive capacities. These changes are known under the concept of epithelial-mesenchymal transition (EMT). EMT is characterized by an upregulation of certain transcription factors, including SNAIL1, which represses genes that are characteristic of an epithelial phenotype, including E-cadherin, and indirectly increase the expression levels of genes, which are associated with the mesenchymal phenotype. It has been suggested that the transcription factor, SNAIL1, decreases the proliferation and increases the migratory and invasive capacities of PCa cell lines. The present study was performed using LNCaP and PC3 cell lines, in which the expression levels of SNAIL1 were increased or silenced through the use of lentiviral vectors. The expression levels of EMT markers were quantified using reverse transcription-quantitative polymerase chain reaction and western blot analysis. In addition, cell survival was analyzed using an MTS assay; cell proliferation was examined using an antibody targeting Ki-67; migration on plates with $8 \mu \mathrm{m}$ pores to allow the passage of cells; and invasiveness was analyzed using a membrane chamber covered in dried basement membrane matrix solution. The levels of apoptosis were determined using a Caspase $3 / 7$ assay containing a substrate modified by caspases 3 and 7. The results demonstrated that the overexpression and silencing of SNAIL1 decreased cell proliferation and survival. However, the overexpression of SNAIL1 decreased apoptosis, compared with cells with the
\end{abstract}

Correspondence to: Dr Héctor R. Contreras, Physiology and Biophysics Program, Institute of Biomedical Sciences, Faculty of Medicine, University of Chile, Clasificador 7, Santiago 8389100 , Chile

E-mail: hcontrer@med.uchile.cl

Key words: snail, epithelial mesenchymal transition, migration, invasion, prostate cancer
SNAIL1-silenced cells, in which cell apoptosis increased. The migration and invasive capacities increased in the cells overexpressing SNAIL1, and decreased when SNAIL1 was silenced. In conclusion, PCa cells overexpressing SNAIL1 exhibited characteristics of an EMT phenotype, whereas the silencing of the SNAIL1 transcriptional repressor promoted an epithelial-like phenotype, with decreased migration and invasion, characteristic of mesenchymal cells.

\section{Introduction}

The predominant diseases affecting the prostate gland are prostatitis, resulting from inflammation by infection; benign prostatic hyperplasia, which develops in the transition zone; and prostate cancer (PCa), which occurs predominantly in the peripheral area (1). It has been established that PCa develops from prostatic intra-epithelial neoplasia, and is preferentially located in the peripheral zone of the prostate $(1,2)$.

The heterogeneity of PCa is important as it allows the distinction between latent and clinical stages, as well as the progression of PCa. The progression of PCa involves de-differentiation of epithelial cells, which lose their organization and adopt a migratory and invasive phenotype $(1,3)$.

Epithelial mesenchymal transition (EMT) is a biological process, which allows polarized epithelial cell interacting with the basal lamina to initiate biochemical changes, which result in a mesenchymal phenotype (4). Among the most important changes are increases in the migration capacity, invasiveness, resistance to apoptosis and production of extracellular matrix (ECM) components (4).

Different molecular mechanisms are involved the initiation of EMT, including the activation of transcription factors, expression of specific cell surface proteins, rearrangement and expression of cytoskeleton proteins, production of ECM-degrading enzymes, including metalloproteinases (MMPs), and changes in the expression of specific microRNAs (miRNAs; miRs) (5).

The physiological and morphogenetic changes that characterize EMT, including decreased expression levels of epithelial genes, such as E-cadherin/cadherin 1, and increased expression levels of mesenchymal genes, such as vimentin, are regulated by transcriptional regulators belonging to the SNAIL (SNAIL1, SLUG and SMUC), zinc finger E-box binding homeobox (ZEB) and TWIST families (5). These changes are 
associated with variation in epithelial cell morphology (loss of apical-basal polarity), to a migratory phenotype $(5,6)$.

Inducing stimuli in the tumor microenvironment, including hypoxia, and various growth factors, including transforming growth factor $\beta$, fibroblast growth factor and insulin-like growth factor 1 , activate specific intracellular downstream signaling pathways. These signaling pathways, including mitogen-activated protein kinase (MAPK), small mothers against decapentaplegic (Smad), glycogen synthase 3, and nuclear factor- $\kappa \mathrm{B}$ components result in increased activity of the ZEB family of transcriptional repressors, TWIST and SNAIL, which downregulate the mRNA expression levels of E-cadherin and other epithelial cell adhesion proteins. Furthermore, these factors indirectly induce the expression of other mesenchymal proteins (6). Epigenetic mechanisms associated with histone lysine methyltransferase promote EMT, whereas miRNAs, including miR-101 or miR-200, may be involved in the maintenance of the epithelial state (5).

A previous study demonstrated that cancer cells acquire a mesenchymal phenotype and express markers, including $\alpha$-smooth muscle actin ( $\alpha$-SMA), fibroblast-specific protein 1 , vimentin and desmin (7). These cells are predominantly located at the invasive front of primary tumors, and are considered to be the cells that are ultimately involved in the later stages of invasion-metastasis, including intravasation, transport through the circulation, extravasation, micrometastasis formation and the establishment of macrometastasis $(4,7)$. The induction of EMT is an important process in the progression of metastatic carcinomas $(7,8)$.

It has been reported that the onset of EMT is dependent on a number of networks of intracellular mechanisms involving the signaling proteins, extracellular signal-regulated kinase, MAPK, phosphoinositide 3-kinase, Akt, Smads, Ras homolog family member $\mathrm{B}, \beta$-catenin, factor enhancer binding lymphoid, Ras and c-Fos, and the cell surface proteins, $\beta_{4}$ integrin, $\alpha_{5} \beta_{1}$ and $\alpha_{V} \beta_{6}(9)$. Activation of EMT is also facilitated by the disruption of cell-cell adhesion junctions and cell-mediated ECM integrins (4).

In patients with castration-resistant $\mathrm{PCa}$, circulating tumor cells coexpressing epithelial markers (E-cadherin) and mesenchymal markers (vimentin, $\mathrm{N}$-cadherin and $\mathrm{O}$-cadherin) have been identified (10). This suggests the in vivo presence of an intermediate EMT phenotype (10). Another previous study showed that the epithelial marker, E-cadherin, and mesenchymal marker, vimentin, are coexpressed in metastatic prostate tissue, suggesting plasticity between EMT and mesenchymal epithelial transition (MET) in a in vivo context (11).

Previous analyses of gene expression profiles using microarrays determined that SNAIL1 increases, compared with that in normal prostatic epithelium, in metastatic CaP $(12,13)$. In addition, immunohistochemical studies have shown that the expression levels of SNAIL1 increase with the progression of PCa $(14,15)$. The SNAIL1 transcription factor has been associated with advanced stages of PCa and a higher Gleason score $(13,15,16)$. Furthermore, our previous study demonstrated using immunohistochemistry the existence of a direct correlation between elevated expression levels of SNAIL1 and Gleason score (17). In PCa cells, SNAIL1 negatively regulates the expression of the tumor suppressor, mammary serine protease inhibitor, by suppressing the activity of its promoter, which leads to increased cell migration and invasion (16). Similarly, in metastatic PCa cell lines, SNAIL1 suppresses the expression of protein kinase Raf, which has been characterized as a metastasis suppressor protein (13). Furthermore, SNAIL1 decreases cell proliferation by repressing the expression of cyclin D2, and SLUG, another member of the Snail family is a negative regulator of $\mathrm{PCa}$ cell proliferation as it suppresses the expression of cyclin D1 (18).

The present study investigated the effects of the SNAIL1 transcription factor on the proliferative, migratory and invasive capacities of PCa cell lines. This study aimed to determine whether the transcription factor SNAIL1 is important for EMT in prostate cancer cell lines and how it influences in the proliferative, migratory and invasive capacities. Silencing SNAIL1 in LNCaP and PC3 cells led to a MET-like process, increasing epithelial characteristics and decreasing tumor cell migration and invasion. Thus SNAIL1 silencing may be considered as a therapeutic target in metastatic $\mathrm{CaP}$.

\section{Materials and methods}

Cell culture. In the present study, the LNCaP PCa cell line (cat. no. CRL-1740; American Type Culture Collection, Manassas, VA, USA) and PC3 cell line (cat. no. CRL-1435; American Type Culture Collection) were used. The LNCaP and PC3 cell lines were maintained in RPMI and Dulbecco's modified Eagle's medium (DMEM)/F-12 (Thermo Fisher Scientific Inc., Waltham, MA, USA) supplemented with $10 \%$ fetal bovine serum (Mediatech, Manassas, VA, USA), respectively. Transduced cells were selected in culture medium containing $2 \mu \mathrm{g} / \mathrm{ml}$ puromycin (Santa Cruz Biotechnology Inc., Santa Cruz Biotechnology, Inc., Dallas, TX, USA) and incubated at $37^{\circ} \mathrm{C}$ in an atmosphere containing $5 \% \mathrm{CO}_{2}$. For the functional assays, the cells were detached with a solution of $2 \mathrm{mM}$ ethylenediaminetetraacetic acid (Calbiochem; EMD Millipore, Billerica, MA, USA) in $1 \mathrm{X}$ phosphate-buffered saline (PBS).

Cell transduction. Lentiviral particles were obtained from GenTarget, Inc. (San Diego, CA, USA). The overexpression vectors containing short hairpin (sh)RNA for SNAIL1 were designed based on the nucleotide sequence (NM_005985.3) obtained from the GenBank database (http://www.ncbi.nlm. nih.gov/genbank/). Transduction was performed in 6-well plates seeded with $7.5 \times 10^{4} \mathrm{LNCaP}$ or PC3 cells/well. A ViraDuctin $^{\mathrm{TM}}$ Lentivirus Transduction kit (Cell Biolabs, Inc., San Diego, CA, USA), at a multiplicity of infection of three was used to facilitate transduction according to the manufacturer's instructions. The experimental design and vectors used are summarized in Table I.

Total protein extraction and western blot analysis. Cells (400 cells $/ \mathrm{ml}$ ) at $70 \%$ confluence in $10 \mathrm{~mm}$ plates were treated with radioimmunoprecipitation assay lysis buffer, containing $20 \mathrm{mM}$ Tris- $\mathrm{HCl}, 150 \mathrm{mM} \mathrm{NaCl}, 1 \mathrm{mM}$ ethylene glycol tetra-acetic acid, $1 \% \mathrm{NP}-40 \mathrm{v} / \mathrm{v}, 1 \%$ sodium deoxycholate w/v, $2.5 \mathrm{mM} \mathrm{Na}_{3} \mathrm{PO}_{4}, 1 \mathrm{mM} \beta$-glycerophosphate and $1 \mathrm{mM}$ $\mathrm{Na}_{3} \mathrm{VO}_{4}$ ( $\mathrm{pH}$ 7.4), in the presence of protease inhibitors (Roche Diagnostics, Basel, Switzerland; cat. no. 11-836-170-001) at $4^{\circ} \mathrm{C}$. The homogenates were centrifuged at $18,000 \mathrm{x}$ g for 
15 min at $4^{\circ} \mathrm{C}$. The supernatants were subsequently collected and quantified using the standard Bradford method. The protein samples were heated at $96^{\circ} \mathrm{C}$ for $5 \mathrm{~min}$, prior to being separated by $10 \%$ SDS-PAGE with electrophoresis at $100 \mathrm{~V}$ for $3 \mathrm{~h}$. The proteins $(50 \mu \mathrm{g})$ were then transferred onto a nitrocellulose membrane $(0.45 \mu \mathrm{m}$ pore; Bio-Rad Laboratories, Inc., Hercules, CA, USA) at $300 \mathrm{~mA}$ for $1.5 \mathrm{~h}$ at $4^{\circ} \mathrm{C}$. The membrane was blocked for $1 \mathrm{~h}$ at room temperature with $10 \%$ bovine serum albumin (BSA; Sigma-Aldrich, St. Louis, MO, USA) in Tris-buffered saline with $0.1 \% 1 \mathrm{X}$-Tween 20, comprising $100 \mathrm{mM}$ Tris- $\mathrm{HCl}, 0.9 \% \mathrm{NaCl}$ and $0.1 \%$ Tween- 20 (pH 7.5). The membranes were then incubated with the following primary antibodies: Monoclonal rabbit anti-SNAIL1 (Cell Signaling Technology, Inc., Danvers, MA, USA; cat. no. 3879; 1:1,000), mouse monoclonal anti-E-cadherin (1:1,000; BD Biosciences, Franklin Lakes, NJ, USA; cat. no. 610181), mouse monoclonal anti-vimentin (1:500; Abcam, Cambridge, UK; cat. no. ab8978) and rabbit polyclonal anti- $\alpha$-tubulin, which served as a loading control (1:1,000; Santa Cruz Biotechnology, Inc.; cat. no. sc-58668). Incubation with the respective primary antibody was performed overnight at $4^{\circ} \mathrm{C}$ with stirring. Membranes were then washed three times with Tris-buffered saline with Tween-20 $0.05 \%$ for $5 \mathrm{~min}$. Subsequently, the membranes were incubated with the corresponding horseradish peroxidase (HRP)-conjugated polyclonal goat anti-mouse $\operatorname{IgG}(1: 5,000$; cat. no. 115-035-003), anti-rabbit (cat. no. 111-035-003) or polyclonal goat anti rabbit IgG (1:5,000; cat. no. 305-035-045) secondary antibodies (all from Jackson Immunoresearch, Inc., West Grove, PA, USA) for $1 \mathrm{~h}$ at room temperature. HRP enzyme activity was analyzed using enhanced chemiluminescence (Biological Industries Israel Beit-Haemek Ltd., Kibbutz Beit-Haemek, Israel). The membrane was visualized using a fluorescence imaging system (Mini ChemiScope 3400; Clinx Science Instruments Co., Ltd., Shanghai, China). Densitometric analysis of bands was performed with the Gel Pro Analyzer 4.5 (Media Cybernetics, Inc., Rockville, MD, USA).

RNA and reverse transcription-quantitative polymerase chain reaction $(R T-q P C R)$. Cells were lysed with TRIzol (Gibco, Thermo Fisher Scientific, Inc.) for RNA extraction according to the manufacturer's instructions. Briefly, RNA was isolated by phase separation using chloroform, precipitated with isopropanol, washed with ethanol and resuspended in diethylpyrocarbonate-treated water. RNA was quantified using SynergyTM HT Multi-Mode Microplate Reader (Biotek Instruments, Inc., Winooski, VT, USA). From $1 \mu \mathrm{g}$ RNA reverse transcription was performed using the AffinityScript QPCR cDNA Synthesis kit (Agilent Technologies, Santa Clara, CA, USA) according to the manufacturer's instructions. The real-time PCR mix was prepared with SYBR Select Master mix (Applied Biosystems, Thermo Fisher Scientific Inc.). The following forward and reverse primers were used: Forward: 5'-GAGCTGCAGGACTCTAATCCAGAG-3' and reverse: 5'-AGCCTGGAGATCCTTGGCCTCAG-3' for SNAIL1; forward: 5'-GACGATGACTACGCTTCTGC-3' and reverse: 5'-T TGTATCCTCTTCGGCTGG-3' for SDC-1; forward: 5'-GGTGCTCTTCCAGGAACCTC-3' and reverse: 5'-TAAGCGATGGCGCATTGTA-3' for E-cadherin, forward: 5'-GGACAGTTCCTGAGGGATCA-3' and reverse: 5'-GGATTGCCTTCCATGTCTGT-3' for N-cadherin; and
Table I. Lentiviral vectors used for cellular transduction.

\begin{tabular}{ll}
\hline Lentiviral vector & \multicolumn{1}{c}{ Name } \\
\hline pLenti-suCMV (SNAI1)-Rsv & Snail \\
pLenti-suCMV-Rsv & Null \\
pLenti-U6-shRNA (SNAI1)\#1/\#2/\#3-Rsv & shRNA Snail \\
pLenti-U6-shRNA (Control-Neg)-Rsv & shRNA control
\end{tabular}

All vectors were RFP-Puro. shRNA, short hairpin RNA.

forward: 5'-CCATCACCATCTTCCAGGAG-3' and reverse: 5'-CCGCCGAACATCCTGCGGTA-3' for glyceraldehyde 3-phosphate dehydrogenase (housekeeping gene). The PCR reaction conditions were as follows: 1 cycle at $90^{\circ} \mathrm{C}$ for $10 \mathrm{~min}$, followed by 40 cycles of $95^{\circ} \mathrm{C}$ for $15 \mathrm{sec}, 60^{\circ} \mathrm{C}$ for $15 \mathrm{sec}$ and $72^{\circ} \mathrm{C}$ for $15 \mathrm{sec}$; and $1 \mathrm{cycle}$ of $95^{\circ} \mathrm{C}$ for $15 \mathrm{sec}, 60^{\circ} \mathrm{C}$ for $15 \mathrm{sec}$ and $95^{\circ} \mathrm{C}$ for $15 \mathrm{sec}$. The experiments were performed in triplicate for each sample. The relative quantification in gene expression was determined using the $2^{-\Delta \Delta \mathrm{Cq}}$ method (19) and the fold changes in gene expression were normalized to a housekeeping gene.

Cell proliferation analysis based on Ki-67 marker. To determine cell proliferation, two silanized slides, which had been subjected to ultraviolet radiation (1300 Series Class II; Thermo Fisher Scientific, Inc.)for $30 \mathrm{~min}$ per side, were each placed on a $100 \mathrm{~mm}$ plate. A total of $1 \times 10^{6}$ cells were subsequently plated and cultured for $24 \mathrm{~h}$ under the same conditions described in the above method. Endogenous peroxidase activity was inhibited using a solution of hydrogen peroxide in $2 \%$ methanol for $30 \mathrm{~min}$. The samples were subsequently washed three times with 1 X PBS, blocked with a solution of $1 \%$ BSA in PBS, and finally incubated overnight with anti-Ki67 (cat. no. M7240; Dako, Glostrup, Denmark) at $4^{\circ} \mathrm{C}$. The following day the samples were washed three times with $1 \mathrm{X}$ PBS, prior to staining using a Vectastain Elite ABC kit (Vector Laboratories, Inc., Burlingame, CA, USA; cat. no. PK-7200). The samples were washed again with $1 \mathrm{X}$ PBS and incubated for 2 min with the DAB substrate (Invitrogen DAB-Plus kit (Thermo Fisher Scientific, Inc.; cat. no.00-2020). Finally, the nuclei were counterstained with hematoxylin (Cell Marque; Sigma-Aldrich) and the samples were observed at a magnification of x400 using a Leica DM-2500 microscope (Leica Microsystems GmbH). The percentage of cells positive for Ki-67 was calculated by counting the number of stained cells (brown) and number of total cells in five randomly-selected fields.

Cell survival assay. A Kit $96{ }^{\circledR}$ CellTiter Aqueous One Solution Cell Proliferation assay (Promega Corporation, Madison, WI, USA) was used for cell survival analysis. A total of $1.5 \times 10^{4}$ cells/well were plated in a 96-well plate. Measurements were performed after $24 \mathrm{~h}$. The MTS in the kit is reduced to formazan (colored) by dehydrogenases of metabolically active cells. The quantity of formazan, determined by absorbance at $490 \mathrm{~nm}$, is proportional to the number of living cells in the culture. The absorbance was measured using a Synergy ${ }^{\mathrm{TM}}$ HT Multi-Mode microplate reader (Bioteke Corporation, Beijing, 
A LNCaP
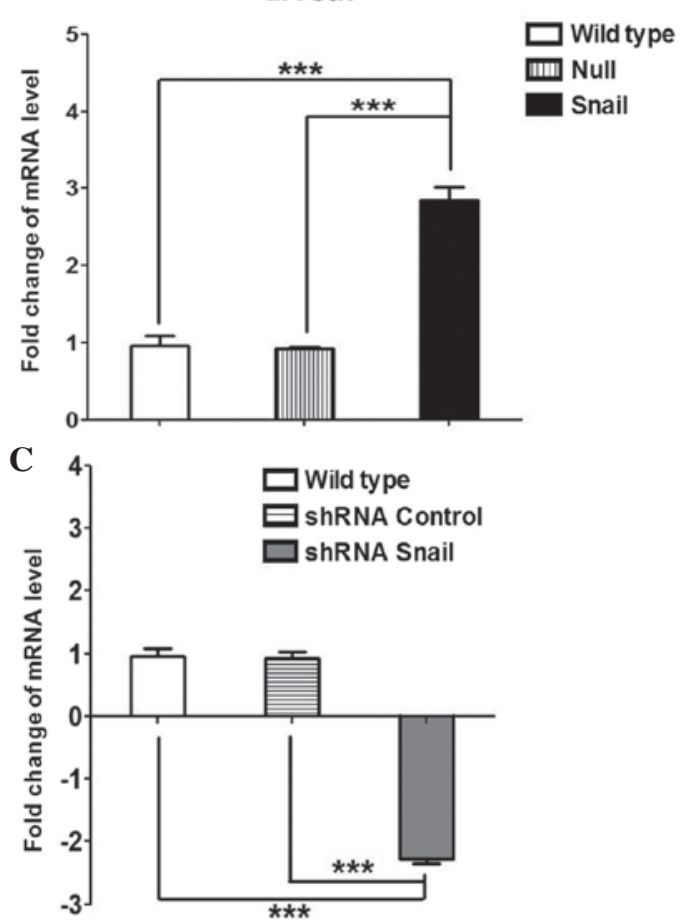

B

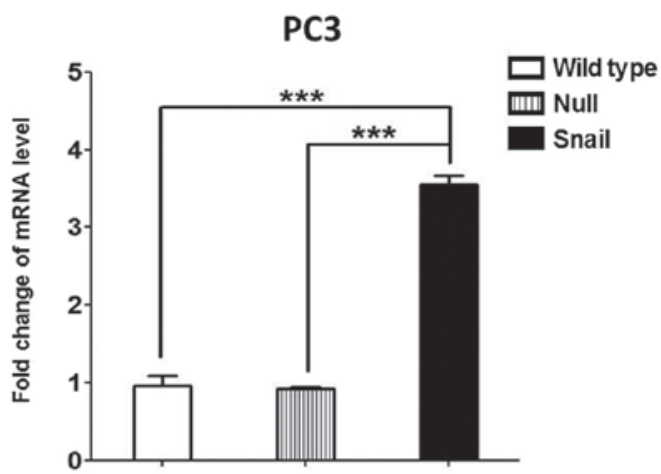

D

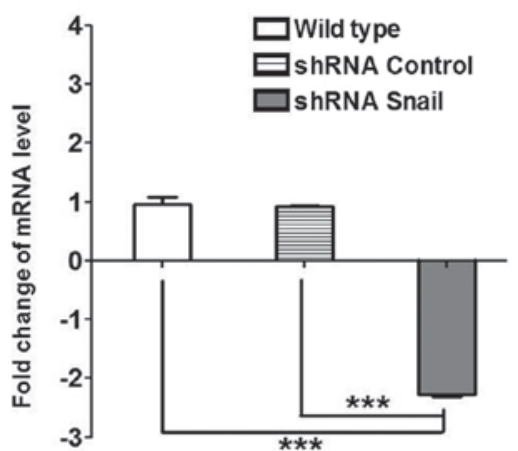

Figure 1. Fold changes in the mRNA expression levels of SNAIL1 in LNCaP and PC3 cells 24 h post-transduction. The colors correspond to wild type (white), null control (vertical lines), Snail (black), shRNA control (horizontal lines) and shRNA Snail (grey) vectors. (A and B) mRNA expression levels of SNAIL1 increased only in the cells transduced with the SNAIL vector. (C and D) mRNA expression levels of SNAIL1 decreased only in the cells transduced with the vector encoding three shRNAs against the coding sequence region of SNAIL1. The data are presented as the fold change in mRNA expression levels of SNAIL1 following normalization to GAPDH and to the untransduced cells. ${ }^{* * *} \mathrm{P}<0.001$. shRNA, short hairpin RNA.

A

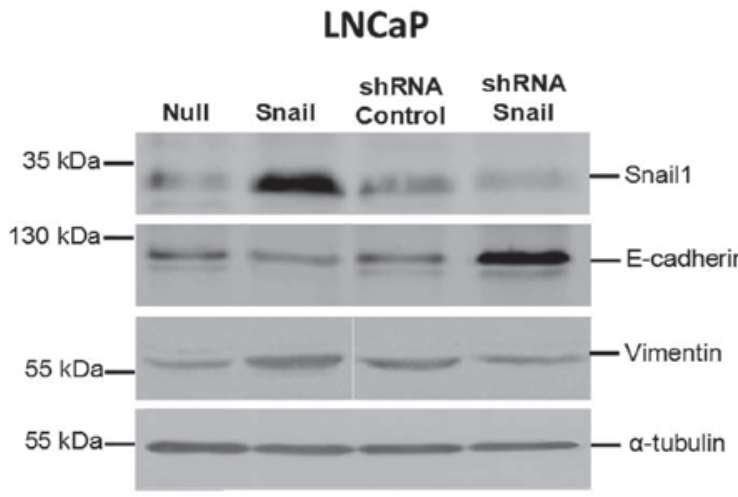

C

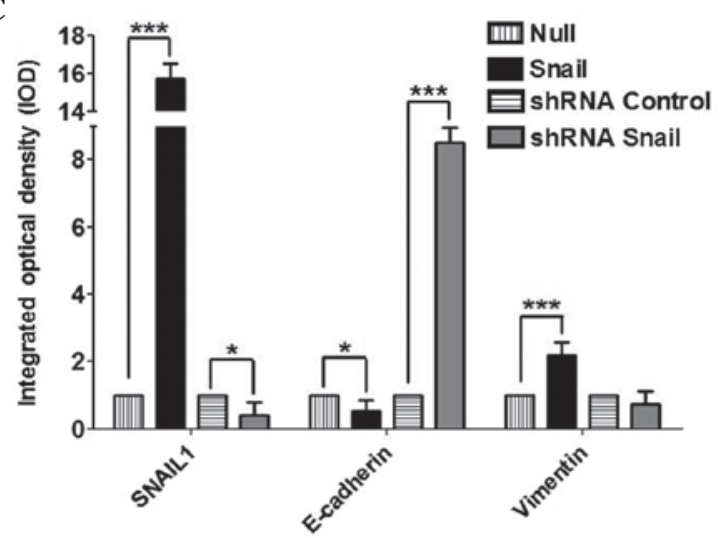

B $\quad$ PC3

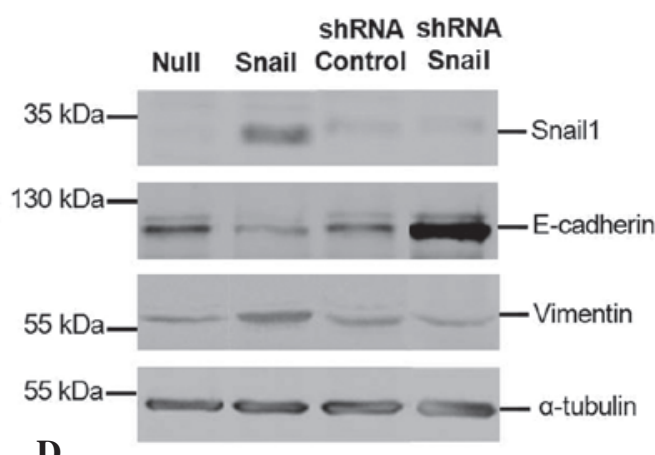

$\mathbf{D}$

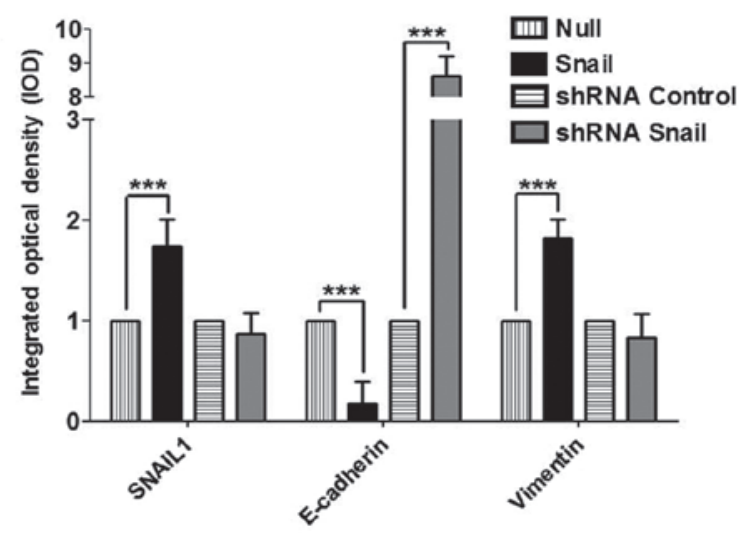

Figure 2. Relative protein expression levels in the LNCaP and PC3 cells. Western blotting was performed to determine the relative protein expression levels of SNAIL1, E-cadherin and vimentin in the (A and B) LNCaP and (C and D) PC3 cells. In both cell lines the overexpression of SNAIL1 upregulated the expression levels of vimentin and downregulated the expression levels of E-cadherin. The cells with SNAIL1 silencing also exhibited upregulated expression levels of E-cadherin. The protein expression levels were normalized to $\alpha$-tubulin. ${ }^{*} \mathrm{P}<0.05$ and ${ }^{* * * *} \mathrm{P}<0.001$. shRNA, short hairpin RNA. 
A

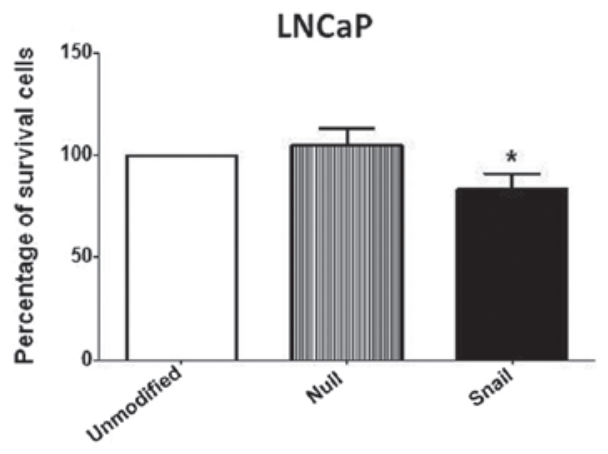

C

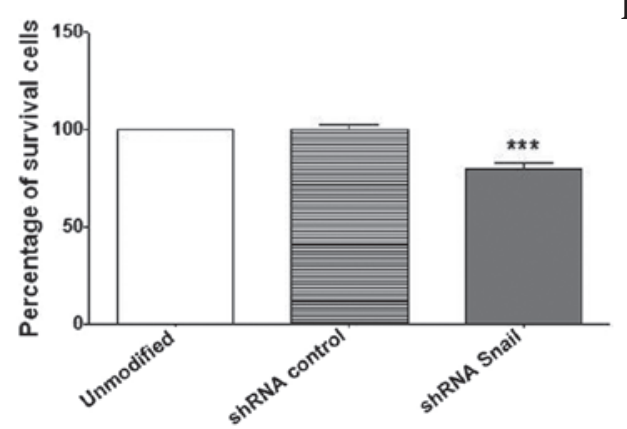

B

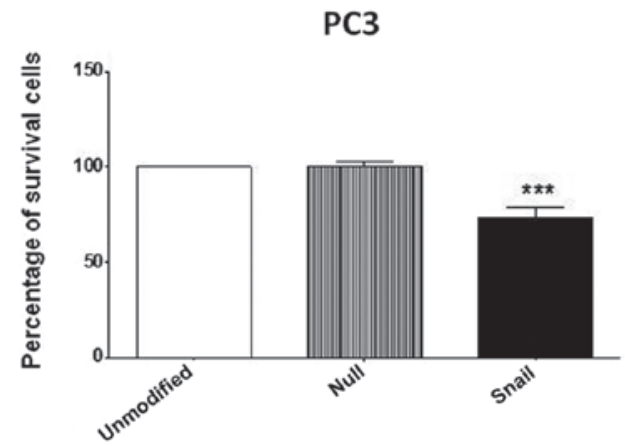

D

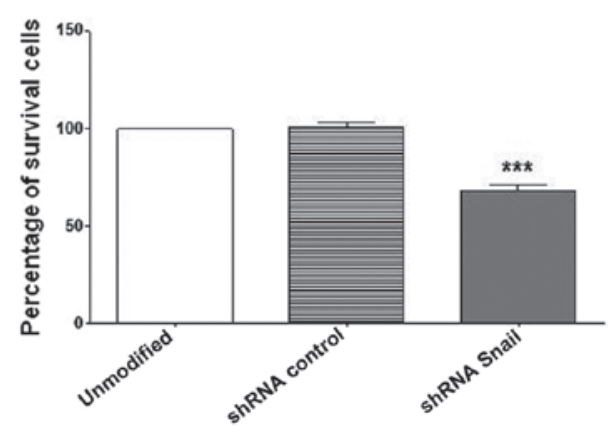

Figure 3. Survival percentage of LNCaP and PC3 cells overexpressing SNAIL1 or with SNAIL1 silencing. The overexpression of SNAIL1 caused a significant decrease in the survival of the (A) LNCap and (B) PC3 cells. Similarly silencing of SNAIL1 decreased the survival of the (C) LNCaP and (D) PC3 cells. Data were normalized to the untransduced cells. ${ }^{*} \mathrm{P}<0.05$ and ${ }^{* * *} \mathrm{P}<0.001$ compared with the corresponding control (null or shRNA control). Values are expressed as the mean \pm standard deviation. shRNA, short hairpin RNA.

China). The absorbance of the untransduced was considered as $100 \%$ survival.

Apoptosis assay. A total of $1.5 \times 10^{4}$ cells of the two cell lines, with SNAIL1 overexpression and silencing, were seeded on a white 96-well plate. The activities of capases 3 and 7 after $24 \mathrm{~h}$ were measured using an Apo-ONE ${ }^{\circledR}$ Kit Homogeneous Caspase-3/7 assay (Promega Corporation). Fluorescence was detected using a Synergy ${ }^{\mathrm{TM}}$ HT Multi-Mode microplate reader (Bioteke Corporation). The data were normalized to control non-transduced cells.

Cell migration and invasion assay. A cell migration and invasion assay was performed using a CytoSelect kit ${ }^{\mathrm{TM}}$ (Cell Biolabs, Inc.), according to the manufacturer's protocol. The polycarbonate wells contained membranes with pores $8 \mu \mathrm{m}$ in size to allow cell migration. The plates used to assess invasiveness also contained membranes with pores $8 \mu \mathrm{m}$ in size, and were coated with basement membrane. The cells $\left(5 \times 10^{4}\right.$ cells/well) were allowed to migrate and invade for $24 \mathrm{~h}$. Each assay was performed in triplicate. Detection of the cells was based on relative fluorescence units. Fluorescence was detected using a Synergy ${ }^{\mathrm{TM}}$ HT Multi-Mode microplate reader (Bioteke Corporation). The data were normalized to the untransduced control cells.

Statistical analysis. The results of the present study were analyzed using a one-way analysis of variance and Dunn's post hoc test. Results are presented as the mean \pm standard deviation. Statistical evaluation of the data was performed using GraphPad Prism software 6.0 (GraphPad Software, Inc., La Jolla, CA, USA). $\mathrm{P}<0.05$ was considered to indicate a statistically significant difference.

\section{Results}

Effects of SNAIL1 on the expression levels of EMT markers in LNCAP and PC3 cells. To determine the effect of SNAIL1 on EMT markers, the LNCaP and PC3 cells were transduced with specific lentiviral vectors. The mRNA expression levels of SNAIL1 increased in the cells transduced with the overexpression vector (Fig. 1A and B), whereas the mRNA expression levels of SNAIL1 decreased in the cells transduced with the silencing vector (Fig. 1C and D). Similar results were observed in the two cell lines.

The effects of SNAIL1 overexpression on the expression levels of epithelial and mesenchymal markers were evaluated using western blot analysis and RT-qPCR. Elevated expression levels of SNAIL1 were associated with a significant decrease in the expression levels of E-cadherin, which may result from the repressor function of SNAIL1 in the nucleus. Furthermore, high expression levels of SNAIL1 significantly increased the expression of vimentin. These results were similar in the LNCaP and PC3 cell lines. (Fig. 2). The same effect was observed in the two cell lines when the mRNA expression levels were evaluated (data not shown). SNAIL1 silencing in the LNCaP and PC3 cells resulted in a significant increase in the expression levels of E-cadherin and decreased expression levels of vimentin (Fig. 2). These data suggested that the overexpression of SNAIL1 induced EMT in the two PCa cell lines, whereas SNAIL1 silencing led to upregulated epithelial gene expression levels.

Following transduction with the SNAIL1-overexpression lentiviral vector, the morphology of the cells gradually became fusiform in shape, similar to fibroblasts, which was accompanied by a loss of cell-cell contact. By contrast, the cells transduced with the empty vector (null) retained their 


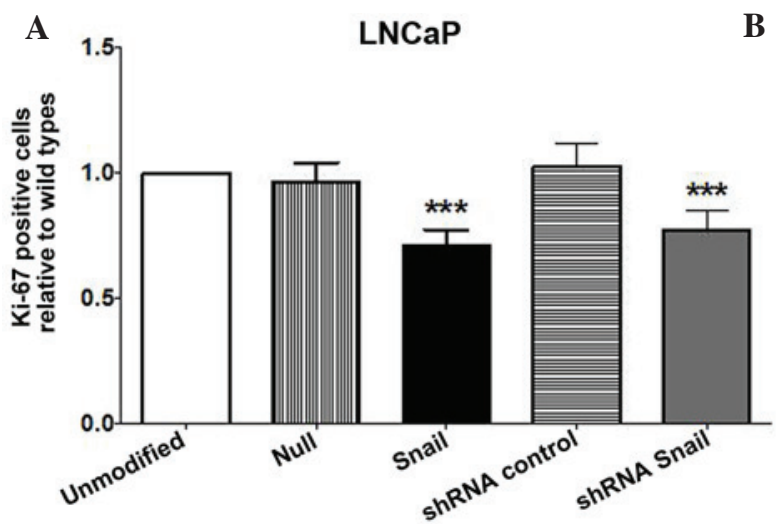

B
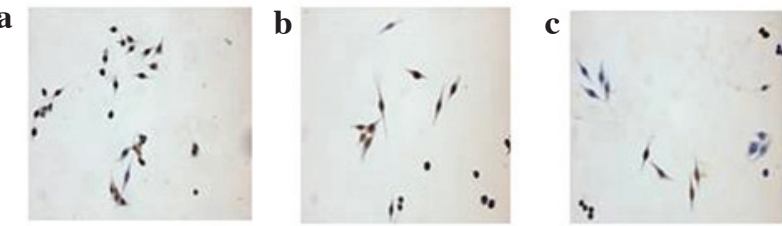

d
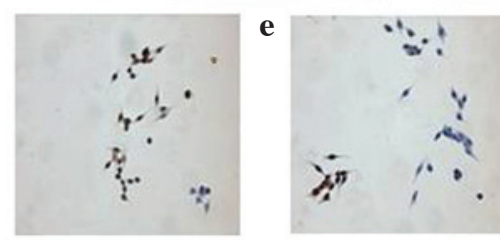

D

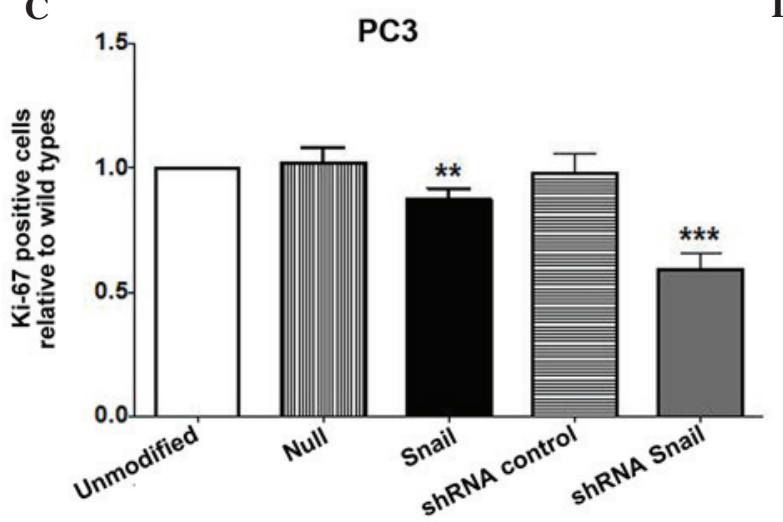

C

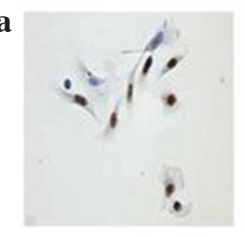

b

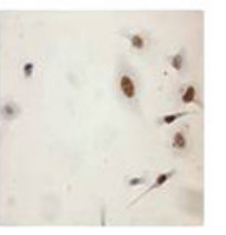

c

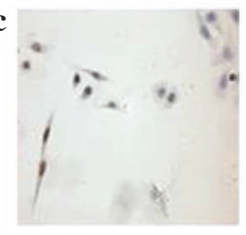

d
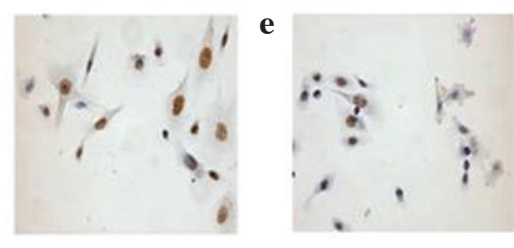

Figure 4. Percentages of LNCaP and PC3 cells positive for Ki-67. Significant decreases in cell proliferation were observed in the (A and B) LNCap and (C and D) PC3 cell lines following overexpression and silencing of SNAIL1. Data were normalized to the untransduced cells. (a) Wild type, (b) null, (c) Snail, (d) shRNA control and (e) shRNA Snail. ${ }^{* *} \mathrm{P}<0.01$ and ${ }^{* * * *} \mathrm{P}<0.001$ compared with the corresponding control (null or shRNA control). Values are expressed as the mean \pm standard deviation. shRNA, short hairpin RNA.

epithelial morphology. These results suggested that overexpression of SNAIL1 in the LNCaP and PC3 cells was sufficient to induce a mesenchymal morphology. By contrast, the PC3 and LNCaP cells transduced with the shRNA-Snail lentiviral vector, causing SNAIL1 mRNA degradation, exhibited an epithelial morphology, and formed compact cluster with apparent cell-cell contact. These changes were more noticeable in the PC3 cells than in the LNCaP cells. The cells transduced with shRNA control maintained their initial morphology (data not shown).

Effects of SNAIL1 on the survival, proliferation and apoptosis of LNCaP and PC3 cells. In the two cell lines, SNAIL1 overexpression significantly decreased cell survival (Fig. 3). The effect of SNAIL1 overexpression and silencing on cell proliferation was also evaluated by examining the expression levels of Ki-67 (Fig. 4). SNAIL1 significantly decreased the levels of these two parameters in the LNCaP and PC3 cells.

Furthermore, assessment of apoptosis was performed by detecting the activity levels of caspases 3 and 7 (Fig. 5). The results demonstrated a decrease in the rate of cell apoptosis following SNAIL1 overexpression. However, cells with SNAIL1 silencing (shRNA-snail) exhibited increases apoptosis.

Effects of SNAIL1 on the migration and invasive capacities of the LNCAP and PC3 cells. In the cells overexpressing SNAIL1, a significant increase in cell migration was observed.
This effect was reversed in the cells treated with shRNA Snail (Fig. 6). Similar effects were observed between the LNCaP and PC3 cells. Furthermore, a cell invasion assay (Fig. 7) revealed that the cells overexpressing SNAIL1 exhibited a significant increase in cell invasion, whereas SNAIL1 silencing significantly reduced the invasive capacity of the two cell lines.

\section{Discussion}

The results of the present study demonstrated the effects of overexpression and silencing of the SNAIL1 transcription factor on certain functional properties of $\mathrm{LNCaP}$ and PC3 cells. The data suggested that the overexpression of SNAIL1 in cell lines induced morphological changes from an epithelial phenotype to a fibroblastoid phenotype, decreased the expression levels of E-cadherin and increased those of vimentin, increased migration and invasion, and decreased proliferation, survival and apoptosis. These changes are closely associated with the EMT. Furthermore, the results of the present study were concordant with those of a previous study by Li et al (20), in which the overexpression of SNAIL1 in retinal pigment epithelium cells resulted in decreased expression levels of the epithelial marker, E-cadherin, and increased the expression levels of the fibronectin and $\alpha$-SMA mesenchymal markers. In addition to these morphological changes, migration also increased in the cells overexpressing SNAIL1.

By contrast, silencing of the transcriptional repressor, SNAIL1, using shRNA Snail cells resulted in an epithelial 


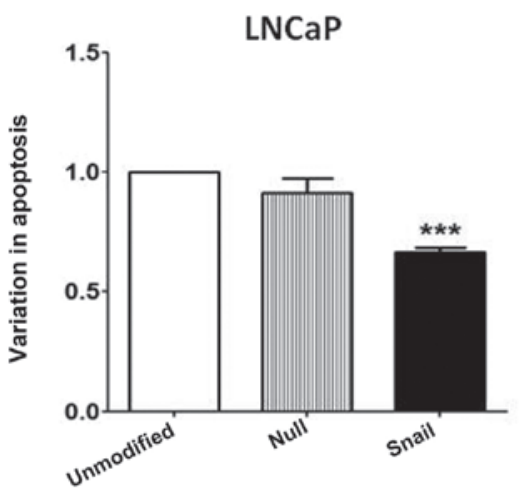

C

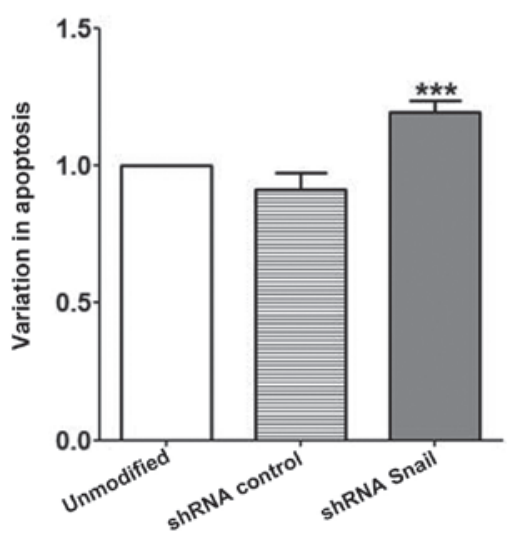

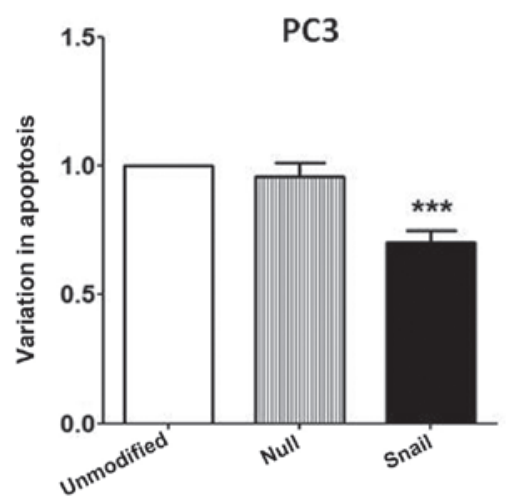

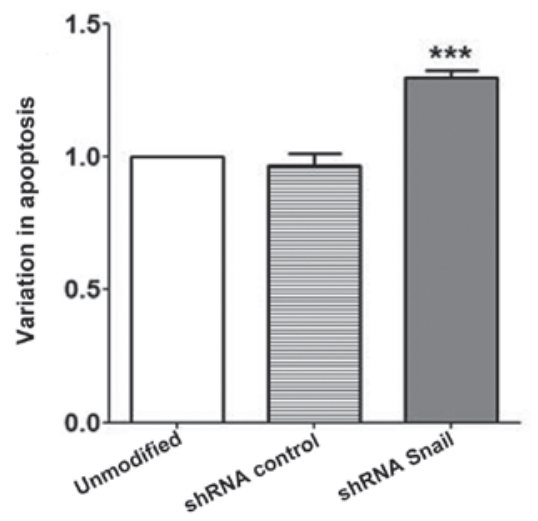

Figure 5. Changes in apoptosis in the LNCaP and PC3 cells at $24 \mathrm{~h}$. The (A) LNCAP and (B) PC3 cell lines with SNAIL1 overexpression exhibited decreased levels of apoptosis, however, the (C) LNCAP and (D) PC3 cells with SNAIL1 silencing exhibited significantly increased levels of apoptosis. Data were normalized to the untransduced cells. ${ }^{* * *} \mathrm{P}<0.001$ compared with the corresponding control (null or shRNA control). Values are expressed as the mean \pm standard deviation. shRNA, short hairpin RNA.

A
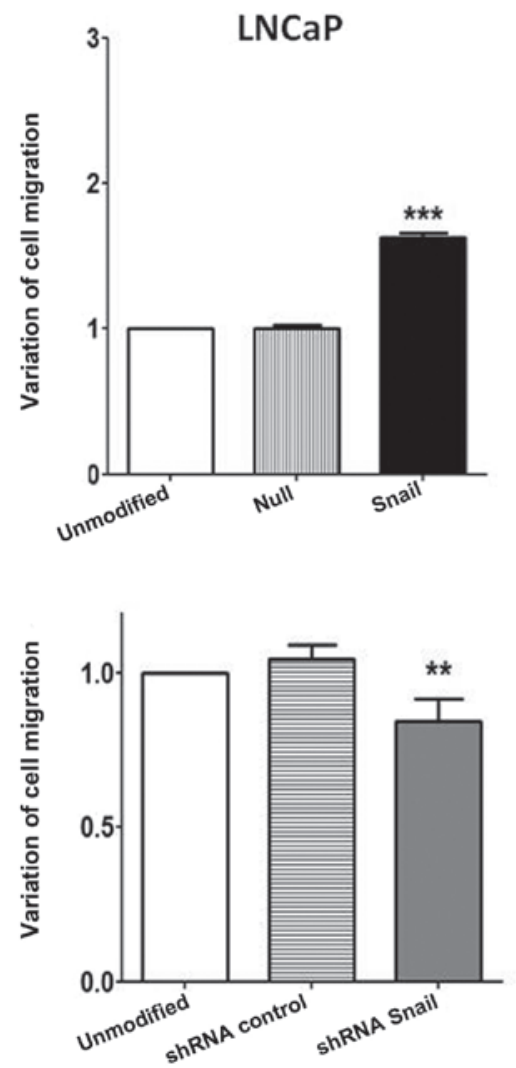

B

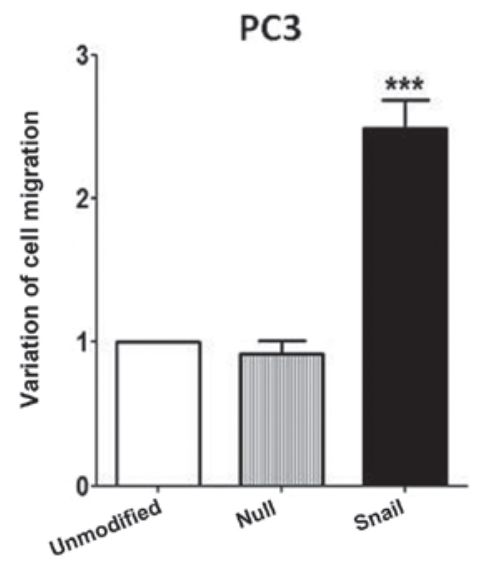

D

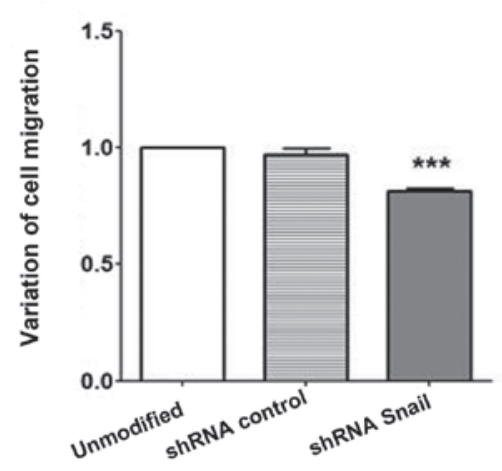

Figure 6. Changes in migration of the LNCaP and PC3 cells at $24 \mathrm{~h}$. The (A) LNCAP and (B) PC3 cell lines with SNAIL1 overexpression exhibited increased levels of migration, however (C) LNCAP and (D) PC3 cells with SNAIL1 silencing exhibited significant decreases in the levels of migration. Data were normalized to the untransduced cells. ${ }^{* *} \mathrm{P}<0,01$ and ${ }^{* * * *} \mathrm{P}<0.001$ compared with the corresponding control (null or shRNA control). Values are expressed as the mean \pm standard deviation. shRNA, short hairpin RNA. 
A

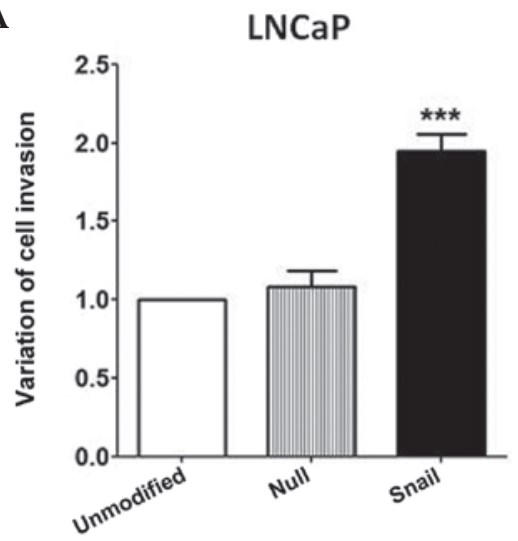

C

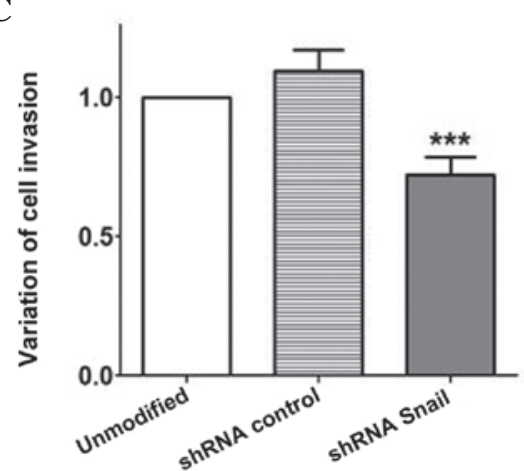

B

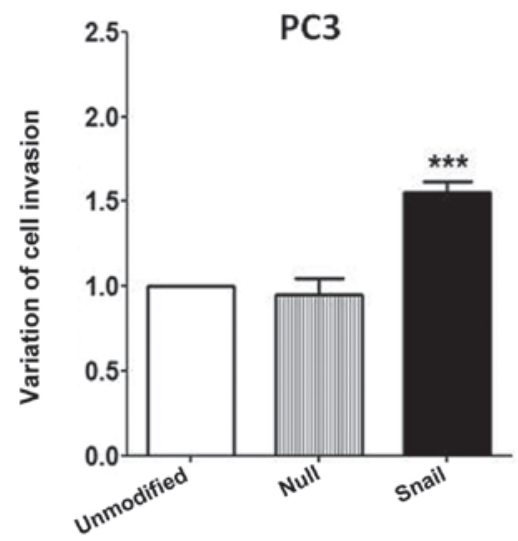

D

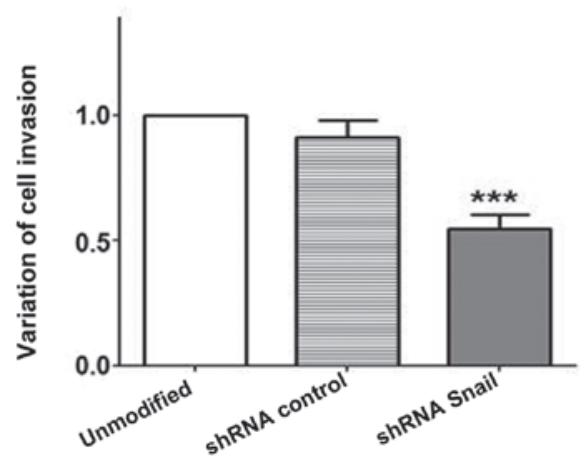

Figure 7. Changes in invasion capacity of the LNCaP and PC3 cells at $24 \mathrm{~h}$. The (A) LNCAP and (B) PC3 cell lines with SNAIL1 overexpression exhibited increased invasion capacity, however the (C) LNCAP and (D) PC3 cells with SNAIL1 silencing exhibited significantly decreased invasion capacity. Data were normalized to the untransduced cells ${ }^{* * *} \mathrm{P}<0.001$ compared with the corresponding control (null or shRNA control). Values are expressed as the mean \pm standard deviation. shRNA, short hairpin RNA.

phenotype, with increased expression levels of E-cadherin and decreased expression levels of vimentin. The in vitro growth of these cells was in the form of clusters, relative to the null and SNAIL1-overexpressing cells. The silencing of SNAIL1 led to cells exhibiting decreased proliferation, survival, migration and invasion, but an increase in apoptosis. These results were concordant with those reported by Olmeda et al (21), which demonstrated that SNAIL1 silencing in Madin-Darby canine kidney epithelial cells by RNA interference resulted in increased expression levels of E-cadherin, decreased expression levels of mesenchymal markers and the inhibition of invasion. Furthermore, it has been reported that the stable silencing of endogenous SNAIL1 in two carcinoma cell lines led to a marked reduction in tumor growth in vivo, accompanied by an increase in tumor differentiation and a significant decrease in the expression levels of MMP-9, and markers of angiogenesis and invasiveness (21).

The changes in cell morphology induced by SNAIL1 observed in the present study were concordant with those reported by Li et al (20). These changes from an epithelial to a mesenchymal phenotype were induced by SNAIL1 overexpression. Li et al (20) reported that retinal pigment epithelial cells change from an epithelial morphology to a fibroblast-like morphology following SNAIL1 overexpression. By contrast, silencing of SNAIL1 induced an epithelial morphology, with growth clusters that differed from those of the control cells, and the absence of individual cell growth. Similar changes were reported in a study by Olmeda et al (21), which demonstrated that MDCK cells with SNAIL1 silencing exhibited an epithelial morphology.

A previous study reported that Snail is involved in epigenetic CpG DNA methylation of the miR-200 loci, which is important for the maintenance of the mesenchymal phenotype in MDCK cells (22). SNAIL1 binds to consensus sequences, termed E-boxes, which are present in the E-cadherin promoter, thereby suppressing expression at the transcriptional level. E-cadherin is a calcium-dependent cell adhesion molecule, and is critical in the maintenance of the epithelial phenotype (23). Decreased expression levels of E-cadherin is considered to be a characteristic marker of EMT (23). The results of the present study revealed that SNAIL1 overexpression decreased the expression of E-cadherin in the LNCaP and PC3 cells. These results suggested that SNAIL1 altered cell morphology and growth by decreasing the expression levels of intercellular junctions and molecules, including E-cadherin. In addition, cells, which initiate EMT also increase their synthesis of cytoskeletal proteins, including vimentin (24). In the present study, the overexpression of SNAIL1 increased the expression levels of the mesenchymal markers, vimentin, in the LNCaP and PC3 cells. These data indicated that the overexpression of SNAIL1 induced EMT in the two cell lines in vitro.

During EMT, epithelial cells have a mesenchymal phenotype, but they also acquire novel functional properties. The results of the present study demonstrated that overexpression of SNAIL1 significantly increased the ability of cells to migrate and invade. These data were concordant with a similar study, 
in which an increase in the migratory and invasive abilities of the PCa cell lines was observed with SNAIL1 overexpression (25). In addition, cells with SNAIL1 silencing exhibited decreased migration and invasion capabilities, indicating that this transcription factor was involved in promoting the metastatic characteristics of migration and invasion.

SNAIL1 regulates $\mathrm{G}_{1}$ transition (early to late) and the $\mathrm{G}_{1} / \mathrm{S}$ checkpoint, and the transcriptional suppression of cyclin D2 and increased expression levels of p21 result in the decreased proliferation of SNAIL1-overexpressing cells (26). However, pro-apoptotic DNA fragmentation factor 40, BH3-interacting domain death agonist, TP53 and caspase 6 genes are repressed by SNAIL1, which provides the cell with apoptosis resistance (27). By contrast, it has been reported that SNAIL1 silencing increases the sensitivity of cells to the DNA damage associated with increased expression levels of pro-apoptotic factors, identified as SNAIL1 targets (28). In addition, SNAIL1 increases the promoter activity of pyruvate dehydrogenase kinase 1 , which phosphorylates pyruvate dehydrogenase, thereby decreasing its activity and the entry of pyruvate into the Krebs cycle (28). It has also been reported that cells overexpressing SNAIL1 exhibit a decrease in the enzymatic activity of Krebs cycle components, including aconitase 2 , isocitrate dehydrogenase 2 and succinate dehydrogenase, and the electron transport chain (Complex II and IV) (28). This mechanism explains the decreased survival of $\mathrm{CaP}$ cell lines overexpressing SNAIL1.

In conclusion, the results of the present study demonstrated that the SNAIL1 transcription factor id important in the induction and phenotypic reversion of EMT in PCa cells. SNAIL1 may serve as a potential therapeutic target to decrease the metastasis of PCa cells. Which anti-apoptotic genes increase following SNAIL1 silencing requires elucidation, in order to develop novel targets to increase the apoptosis of cancer cells.

\section{Acknowledgements}

The authors of the present study would like to thank Ms. Graciela Caroca for her technical assistance. The present study was supported by grants from the Fondo Nacional de Ciencia y Tecnología [grant nos. 1151214 and 1110269 to Dr Héctor R Contreras, and 1140417 to Dr Enrique A Castellón).

\section{References}

1. Shen MM and Abate-Shen C: Molecular genetics of prostate cancer: New prospects for old challenges. Genes Dev 24: 1967-2000, 2010.

2. De Marzo AM, Platz EA, Sutcliffe S, Xu J, Grönberg H, Drake CG, Nakai Y, Isaacs WB and Nelson WG: Inflammation in prostate carcinogenesis. Nat Rev Cancer 7: 256-269, 2007.

3. De Marzo AM, Nelson WG, Isaacs WB and Epstein JI: Pathological and molecular aspects of prostate cancer. Lancet 361: 955-964, 2003.

4. Kalluri R and Weinberg RA: The basics of epithelial-mesenchymal transition. J Clin Invest 119: 1420-1428, 2009.

5. Nauseef JT and Henry MD: Epithelial-to-mesenchymal transition in prostate cancer: Paradigm or puzzle? Nat Rev Urol 8: 428-439, 2011.

6. Gavert N and Ben-Ze'ev A: Epithelial-mesenchymal transition and the invasive potential of tumors. Trends Mol Med 14: 199-209, 2008

7. Thiery JP: Epithelial-mesenchymal transitions in tumour progression. Nat Rev Cancer 2: 442-454, 2002.
8. Thiery JP, Acloque H, Huang RY and Nieto MA: Epithelial-mesenchymal transitions in development and disease. Cell 139: 871-90, 2009.

9. Tse JC and Kalluri R: Mechanisms of metastasis: Epithelial-to-mesenchymal transition and contribution of tumor microenvironment. J Cell Biochem 101: 816-829, 2007.

10. Armstrong AJ, Marengo MS, Oltean S, Kemeny G, Bitting RL, Turnbull JD, Herold CI, Marcom PK, George DJ and Garcia-Blanco MA: Circulating tumor cells from patients with advanced prostate and breast cancer display both epithelial and mesenchymal markers. Mol Cancer Res 9: 997-1007, 2011.

11. Chao Y, Wu Q, Acquafondata M, Dhir R and Wells A: Partial mesenchymal to epithelial reverting transition in breast and prostate cancer metastases. Cancer Microenviron 5: 19-28, 2012.

12. Dhanasekaran SM, Barrette TR, Ghosh D, Shah R, Varambally $S$, Kurachi K, Pienta KJ, Rubin MA and Chinnaiyan AM: Delineation of prognostic biomarkers in prostate cáncer. Nature 412: 822-826, 2001.

13. Beach S, Tang H, Park S, Dhillon AS, Keller ET, Kolch W and Yeung KC: Snail is a repressor of RKIP transcription in metastatic prostate cancer cells. Oncogene 27: 2243-2248, 2008.

14. Odero-Marah VA, Wang R, Chu G, Zayzafoon M, Xu J, Shi C, Marshall FF, Zhau HE and Chung LW: Receptor activator of NF-kappaB Ligand (RANKL) expression is associated with epithelial to mesenchymal transition in human prostate cancer cells. Cell Res 18: 858-870, 2008.

15. Heebøll S, Borre M, Ottosen PD, Dyrskjøt L, Orntoft TF and Tørring N: Snaill is over-expressed in prostate cancer. APMIS 117: 196-204, 2009.

16. Neal CL, Henderson V, Smith BN, McKeithen D, Graham T, Vo BT and Odero-Marah VA: Snail transcription factor negatively regulates maspin tumor suppressor in human prostate cancer cells. BMC Cancer 12: 336, 2012.

17. Poblete CE, Fulla J, Gallardo M, Muñoz V, Castellón EA, Gallegos I and Contreras HR: Increased SNAIL expression and low syndecan levels are associated with high Gleason grade in prostate cancer. Int J Oncol 44: 647-654, 2012.

18. Liu J, Uygur B, Zhang Z, Shao L, Romero D, Vary C, Ding Q and Wu WS: Slug inhibits proliferation of human prostate cancer cells via downregulation of cyclin D1 expression. Prostate 70: 1768-1777, 2010.

19. Livak KJ and Schmittgen TD: Analysis of relative gene expression data using real-time quantitative PCR and the $2-\Delta \Delta \mathrm{Ct}$ method. Methods 25: 402-408, 2001.

20. Li H, Li M, Xu D, Zhao C, Liu G and Wang F: Overexpression of Snail in retinal pigment epithelial triggered epithelial-mesenchymal transition. Biochem Biophys Res Commun 446: 347-351, 2014.

21. Olmeda D, Jordá M, Peinado H, Fabra A and Cano A: Snail silencing effectively suppresses tumour growth and invasiveness. Oncogene 26: 1862-1874, 2007.

22. Díaz-López A, Díaz-Martinez A, Moreno-Bueno G, Cuevas EP, Santos V, Olmeda D, Portillo F, Palacios J and Cano A: Zeb1 and Snaill engage miR-200f transcriptional and epigenetic regulation during EMT. Int J Cancer 136: E62-E73, 2015.

23. Cano A, Pérez-Moreno MA, Rodrigo I, Locascio A, Blanco MJ, del Barrio MG, Portillo F and Nieto MA: The transcription factor snail controls epithelial-mesenchymal transitions by repressing E-cadherin expression. Nat Cell Biol 2: 76-83, 2002.

24. Zeisberg M and Neilson EG: Biomarkers for epithelial-mesenchymal transitions. J Clin Invest 119: 1429-1437, 2009.

25. Neal CL, McKeithen D and Odero-Marah VA: Snail negatively regulates cell adhesion to extracellular matrix and integrin expression via the MAPK pathway in prostate cancer cells. Cell Adh Migr 5: 249-257, 2011.

26. Vega S, Morales AV, Ocaña OH, Valdés F, Fabregat I and Nieto MA: Snail blocks the cell cycle and confers resistance to cell death. Genes Dev 18: 1131-1143, 2004.

27. Kajita M, McClinic KN and Wade PA: Aberrant expression of the transcription factors snail and slug alters the response to genotoxic stress. Mol Cell Biol 24: 7559-7566, 2004.

28. Haraguchi M, Indo HP, Iwasaki Y, Iwashita Y, Fukushige T, Majima HJ, Izumo K, Horiuchi M, Kanekura T, Furukawa T and Ozawa M: Snail modulates cell metabolism in MDCK cells. Biochem Biophys Res Commun 432: 618-625, 2013. 